\title{
Clima escolar y tipología docente: la violencia escolar en las prácticas educativas
}

\author{
School climate and teacher typology: \\ school violence in educational practices
}

Jorge Manuel Rojas Bravo*

\section{Resumen}

La violencia escolar actualmente es una temática de preocupación pública y privada. En este sentido, en Chile se han organizado una serie de seminarios, propuestas y acciones en torno a la modificación de este problema sociológico con tintes de problema social. Sin embargo, el paradigma que ha primado en la observación del problema se ha sobre-enfocado en los aspectos individuales: "los estudiantes abusivos" o su contrapartida, "la víctima indefensa". Sin duda que esta mirada es cierta, pero no podemos ver el problema sólo desde esta perspectiva. De hecho en los análisis sobre las determinantes del nacimiento de la violencia y sus manifestaciones, no sólo la podemos apreciar desde "la familia" o las características personales de los estudiantes, sino que nuestra mirada se dirige hacia la institución escolar misma. La violencia escolar ha estado desde siempre en el sistema educativo, sin embargo, las viejas prácticas de disciplinamiento y control del estudiantado pocas veces se observan como tales. El objetivo principal del estudio es analizar aquellas prácticas educativas de cuatro escuelas primarias, que pueden ser conceptualizadas como violentas, y que precisamente se han invisibilizado como tal. La metodología utilizada fue de corte etnográfica, en la que se utilizaron 160 observaciones participantes y 16 entrevistas semi-estructuradas. Los espacios de observación fueron cuatro salas de clase, principalmente la interacción profesor-alumno. Los principales resultados permiten comprender cómo ciertos aspectos de las dinámicas o estáticas mismas de las prácticas escolares generan espacios de continua violencia tanto implícita como explícita hacia los estudiantes y que los climas que se crean no son los más aptos para el aprendizaje de los valores sociales que promueven nuestras reformas educativas. A partir de esta lógica se logró determinar distintos escenarios y tipologías de docentes que contienen distintas formas de violencia en sus prácticas pedagógicas y los climas de aula que generan. De esta forma, tenemos tres tipos de escenario posibles y tres tipos de docente observados.

PALABRAS CLAVES: violencia escolar, control y disciplinamiento, climas escolares

\section{Abstract}

School violence is currently a matter of public and private concern. In Chile a series of seminars, proposals and actions have been organized around the modification of this sociological issue with overtones of a social problem. Nevertheless, in the observation of the problem, too much emphasis has been put on the paradigm that prioritizes individual aspects of "abusive students" or their counterparts, the "defenseless victims". Although an appropriate paradigm, the problem cannot be viewed through this lens alone. Indeed, the analysis of the causes leading to violence and its manifestations should extend beyond "the family" and the students' personal characteris ICT. Accordingly, the approach taken herein considers the school institution itself. Although school violence has always existed in the educational system, the traditional practices of discipline and control of the student body are rarely seen as violent. In order to visualize the educational practices that can be conceptualized as violent and and have been invisibiilized as such we went into the schools and conducted participant observations and semi-structured 
interviews (an ethnographic, qualitative methodology). Our main results allowed us to understand how certain aspects of the very dynamics (or "staTIC") of specific school practices generate spaces of continuous violence implicit as well as explicit towards the students, and that the climates created in this milieu are not the most apt for learning the social values promoted by our educational reforms. We have determined different scenarios and typologies of teachers (observing three of each) whose pedagogical practices contain different forms of violence and the classroom climates generated by these factors.

KEY WORDS: school violence, control and discipline, school climates

\section{Presentación: de la violencia estudiantil hacia la violencia de la escuela}

El ejercicio del bullying y la violencia escolar en Chile es altamente prevaleciente en nuestros establecimientos escolares. Tanto es así que el prevenir la violencia escolar se ha convertido en una nueva demanda social a la educación (Villalta, Saavedra y Muñoz, 2007). Es importante rescatar el hecho de que esta gran demanda no es necesariamente producto de un aumento desmedido de la violencia en las escuelas, sino que parte de esta apreciación se obtiene desde el cambio de sensibilidad (Gallego y Antón, 2008) que hoy en día tenemos respecto de las formas y conductas que consideramos aceptables. Es así como el hecho de las burlas, golpes y aislamientos, son tomados desde una mirada más amplia y crítica, sobre todo por las derivaciones subjetivas y transubjetivas que producen; es decir, esa idea de que las relaciones basadas en el abuso de poder no son aceptadas porque generan un cierto clima de desigualdad y vulneración de derechos y sobre todo, que no están acordes a los ideales democráticos ni a la cultura de paz que hoy en día se persigue. Acorde con esta nueva sensibilidad, se han realizado muchos estudios que han visibilizado este fenómeno, lo cual nos permite sistematizar y tener una visión más nítida sobre los grados de violencia que nuestro alumnado recibe y ejerce.

En el estudio de la UNESCO/MINEDUC (2005) se señala que el 23\% de los alumnos que asisten a educación primaria (de primer a octavo nivel) sufre violencia en el establecimiento. $\mathrm{Al}$ indagar más profundamente en este fenómeno aparece que sólo el $9 \%$ de los alumnos dice haber sido víctima de agresión física; sin embargo, el 14\% sufre aislamiento social o ignorancia de sus pares, el $23 \%$ afirma haber sufrido robo o daño a sus pertenencias y un $28 \%$ asevera ser víctima frecuente de insultos. Otros actores relevantes dentro de la escena educativa, que igualmente se ven perjudicados, son los docentes. De acuerdo con Mena, Romagnoli y Valdés (2006) un $67 \%$ siente que le faltan el respeto, mientras 14\% señala sufrir agresión física por parte de los alumnos. Adicionalmente, según el Ministerio del Interior (2007), el 36,6\% de los alumnos y el $40,6 \%$ de los docentes mencionan que la frecuencia con que ocurren las agresiones en sus establecimientos es alta.

Numerosos estudios psicológicos y educativos se han realizado en Chile y en el mundo y han provisto de una detallada descripción del bullying y la victimización entre los estudiantes (Yoneyama y Naito, 2003). Como consecuencia de estos masivos antecedentes se han acumulado muchos aspectos como la prevalencia, la frecuencia, la intensidad, duración de la violencia (Ministerio del Interior, 2007; Ministerio de Educación, 2003; UNESCO, 2005), lugar donde ocurre, diferencias de sexo, diferentes formas y métodos de bullying (física, verbal y relacional), rasgos conductuales de los bullies, víctimas y bullies/victimas (Yoneyama y Naito, 2003) y los sentidos atribuidos a la violencia (García y Madriaza, 2005). En fin, una serie de antecedentes que nos permiten tener una apreciación bastante acertada de lo que está pasando actualmente con nuestros estudiantes, además de conocer sus tipos de interacciones y los grados de violencia que sufren. Esto ha dado como resultado conocer muy bien ciertos aspectos del problema, con una gran cantidad de antecedentes empíricos y conceptuales. 


\section{Problema: la escuela como unidad de análisis}

Por otra parte, la explicación del por qué algunos estudiantes hacen bullying ha sido buscada a través del examen de los atributos personales de los bullies y de las víctimas y sus desventajas sociales y/o familiares. De esta manera, se han buscado las explicaciones del bullying y la violencia escolar, principalmente desde una postura más individualista y externa. Individualista por cuanto se centra en ciertas características intrínsecas y particulares de los sujetos las que, muchas veces, son conceptualizadas como aspectos "riesgosos" de los estudiantes; y externa por cuanto observan los climas que esos sujetos tienen, es decir, el hogar, la comunidad, independientes de la vida o clima escolar en el que se encuentran. Hasta ahora, factores de la escuela que han sido correlacionados con la prevalencia de bullying y la victimización han sido escasamente investigados (Farrington, 1993; Wolke et al., 2001; en Yoneyama y Naito, 2003).

Sin embargo, y considerando toda la importancia de investigar los factores de la escuela desde estos puntos de vista, algunas causas del bullying necesitan de mayores visibilizaciones y por ende de investigaciones. En la literatura (sobre todo la nacional) hay una insuficiente atención al contexto escolar, y ella refleja una larga tradición teórica/empírica en el abordaje de la agresión estudiantil en general. Aspectos propios de las instituciones escolares han sido escasamente considerados para explicar los climas escolares resultantes en los distintos colegios donde se pueden observar bullying y violencia escolar. Las dimensiones curriculares, pedagógicas, la estructura organizacional, las relaciones de poder y la legitimación de la autoridad son factores que van gatillando o inhibiendo ciertos "ethos" escolares, que se traducirán en climas rápidamente reconocibles.

Las escuelas son micro mundos o micro sistemas que tienen sus propias lógicas y formas de actuar, que producen sus propias dinámicas o inercias, las cuales van a dar el marco preciso para la existencia o no de altos o bajos niveles de bullying y violencia escolar. De esta manera, la presente investigación ve a la escuela como una institución que, por sí misma, es unidad de análisis. Veremos a la escuela como un agente de socialización que establece ciertas pautas interaccionales e ideacionales en sus miembros o comunidad escolar.

Teniendo en cuenta todas las contribuciones de la sociología de la educación en la comprensión de que las instituciones escolares también pueden ser instituciones que producen y reproducen las condiciones de desigualdad de los sujetos, que el ejercicio del disciplinamiento de los cuerpos y el pensamiento, de la diferenciación/indiferenciación y jerarquización también son procesos presentes, vamos a considerar con más fuerza la posibilidad de entender que los malos climas escolares, que el abuso de poder y las diferenciaciones internas en los establecimientos son producto, también, del cómo se estructura esa escuela en particular. Claros han sido los aportes de Bourdieu y Passeron (2001) con sus conceptos de Habitus y violencia simbólica; de Bernstein (1994) con su idea de los códigos restringidos, enmarcación y clasificación, y la idea del currículo oculto de Torres (1992), los cuales nos han demostrado que la escuela no es una institución que está encargada de entregar conocimientos de forma neutra sino que tiende a producir/reproducir relaciones de poder.

Considerando los puntos anteriores, no todas las escuelas poseen una estructura democrática ni menos aún pueden ser consideradas eficaces (UNESCO, 2008b). De hecho existe una enorme cantidad de escuelas que siguen conservando una estructura interna autoritaria (Solar, 2002, en Guerrero, 2005) donde se sustenta el uso de la agresión (física o verbal), las reglas de convivencia no pasan del reglamento disciplinario. En fin, tenemos actualmente muchas instituciones escolares donde el "bullying es particularmente un resultado de la estructura y organización de la escuela misma” (Askew, 1988, en Yoneyama y Naito, 2003, 69). 
Este cambio de foco no sólo ve cómo la escuela es capaz de producir (de forma implícita) una estructura que favorece, en términos generales, un mal clima de convivencia escolar, sino también, ella misma se ve perjudicada en sus funciones internas. La violencia escolar ejerce un triple impacto en el funcionamiento y funciones de la escuela (Trianas, Sánchez y Muñoz, 2001): desmoraliza y desmotiva laboralmente al profesorado, produce en la institución escolar un abandono de sus objetivos prioritarios de enseñanza, y provoca también el abandono de los objetivos de formación humana del alumnado al centrar la atención en aquellos estudiantes que muestran más problemas de disciplina.

Por lo tanto, se pasa de una visión individualista y directa de la violencia hacia a una visión colectiva y amplia. En este sentido ya no es sólo importante los tipos de violencia que los estudiantes ejercen, sino el cómo la institución escolar, a través de su accionar cotidiano, ejerce cierto tipo de violencia (simbólica, disciplinaria, segregacionista, etc.) que a su vez reproduce malos climas escolares.

Si bien nuestro enfoque observa el cómo la escuela puede ser un agente que tiende a la reproducción de las desigualdades sociales, también consideramos que ésta puede ser una institución que gatille los cambios sociales, es decir, las escuelas también pueden ser vistas como instancias de socialización "positivas" que logren construir y revitalizar muchos ideales asociados a ellas. A través de la evaluación crítica de las prácticas y currículo oculto y el entendimiento de las lógicas internas de las escuelas, éstas pueden muy bien ser un agente de cambio social (Giroux, 1990). Los múltiples ejemplos de las escuelas eficaces (ver UNESCO, 2008b), que albergan estructuras democráticas y excelentes resultados académicos con poblaciones desfavorecidas, nos dan pie para pensar que el develar estas lógicas internas en los colegios tiene la finalidad de evidenciar y con ello, poder iniciar un proceso de cambio que permita instaurar buenas prácticas educativas y organizacionales que se vean reflejadas en la mejora de los climas y culturas escolares, potenciando el respeto, la inclusión y la construcción de una ciudadanía democrática y madura.

\section{Diseño Metodológico}

Los diseños metodológicos y las técnicas específicas seleccionadas en una investigación deben estar acordes y en sintonía con el fenómeno que se plantean estudiar (Vieytes, 2004). En nuestro caso, la problemática del conflicto en las escuelas no es un fenómeno que sólo se exprese como una cuestión estructural, en donde los sujetos no son más que parte de un engranaje mayor. Dentro del espacio de las instituciones escolares se dan en forma conjunta los tres niveles de análisis en que podemos abordar los fenómenos; micro, meso y macro. Nuestra investigación apunta hacia la primera dimensión: el aspecto micro que busca observar todas las interacciones sociales, preferentemente entre el docente y el alumnado que de tensiones y conflictos y un determinado clima de aula.

Ahora bien, las relaciones sociales y pautas de interacción dentro de estos micro mundos no son siempre visibles de forma inmediata: los actores muchas veces no tienen conciencia directa de lo que "aquí está pasando". Es así cómo los conceptos de develamiento y visibilización toman fuerza. Este supuesto epistemológico de la actividad educativa interpreta como la realidad que envuelve a los sujetos no siempre evidencia sino que a través del ejercicio consciente y crítico se le puede observar.

En nuestro caso el método elegido será el estudio de caso, que es definido como un examen intenso de un fenómeno o de ciertas facetas de él (Rodríguez y otros, 1999). Los estudios de caso pueden ser conceptualizados como estudios de caso múltiple (Yin, 1984 en Rodríguez y otros, 1999), ya que las investigaciones pueden abarcar distintas dimensiones de uno o varios 
casos a la vez. En la presente investigación la dimensión que se rescata son las interacciones sociales dentro de las salas de clase de cuatro establecimientos educacionales, los cuales comparten ciertas variables educativas importantes. En este sentido, el hecho de compartir ciertos elementos que los caracterizan y que están presentes en mayor o menor medida en muchos establecimientos educacionales es que son tomados como "muestra" de un aspecto teórico que quiere ser comprendido y explicado. De esta forma los establecimientos escolares elegidos serán conceptualizados bajo la lógica de los estudios de caso instrumentales (Stake, 1994 en Rodríguez y otros, 1999), los cuales nos permitirán una mejor comprensión de la temática de las prácticas educativas y cómo ellas pueden ser una fuente de violencia escolar.

\subsection{Población De Estudio}

Para la selección de los casos de estudio se utilizó la base de datos del Sistema de Medición de Calidad de la Educación (SIMCE), la cual nos arroja el nivel de aprendizajes promedio de las escuelas en diferentes campos disciplinarios, nivel socioeconómico y condición de vulnerabilidad social de los alumnos de cada establecimiento. La tabla $\mathrm{N}^{\circ} 1$ nos detalla las características de las escuelas en el estudio.

\section{Tabla N¹: Características de las escuelas}

Fuente: Ministerio de Educación

\begin{tabular}{|c|l|l|l|l|}
\hline Escuela & Comuna & $\begin{array}{l}\text { Dependencia } \\
\text { administrativa }\end{array}$ & Nivel socioeconómico & $\begin{array}{l}\text { Rendimiento académico } \\
\text { SIMCE }\end{array}$ \\
\hline 1 & Yumbel & Pública (Municipal) & Medio-bajo* & $\begin{array}{l}270 \text { Lenguaje } \\
\text { 271 Matemática } \\
\text { 259 Ciencias sociales }\end{array}$ \\
\hline 2 & $\begin{array}{l}\text { S a n t a } \\
\text { Bárbara }\end{array}$ & Pública (Municipal) & Medio-bajo & $\begin{array}{l}254 \text { Lenguaje } \\
\text { 254 Matemática } \\
216 \text { Ciencias sociales }\end{array}$ \\
\hline 3 & Los & Pública (Municipal) & Medio** & $\begin{array}{l}245 \text { Lenguaje } \\
234 \text { Matemática } \\
\text { 236 Ciencias sociales }\end{array}$ \\
\hline 4 & Ángeles & & & $\begin{array}{l}228 \text { Lenguaje } \\
232 \text { Matemática } \\
217 \text { Ciencias sociales }\end{array}$ \\
\hline
\end{tabular}

*El nivel medio-bajo corresponde a padres con 9 a 10 años de escolaridad, con un ingreso de entre US\$ 375 y US\$ 585 aproximadamente.

El nivel de vulnerabilidad social de la escuela se sitúa entre el $54,01 \%$ y el $80 \% "$.

El nivel medio corresponde a padres con 11 y 12 años de escolaridad, un ingreso de entre US\$ 585 y US\$1.040. El nivel de vulnerabilidad social de la escuela se encuentra entre el $27,01 \%$ y $54 \%$.

Los puntajes SIMCE van desde 0 hasta 350 puntos.

Como es posible apreciar en la tabla $\mathrm{N}^{\circ} 1$, las escuelas escogidas comparten altos niveles de vulnerabilidad y bajos rendimientos académicos como asimismo el ser de carácter público. Este es el contexto definido como importante para la investigación, por cuanto es en este contexto donde se puede desarrollar el disciplinamiento constante. Obviamente, estos casos no implican tendencia sino ejemplos de ciertos tipos de pedagogía y sus posibles climas escolares. Otro aspecto a considerar es que la elección de este primer nivel fue en base a la 
suposición de que es aquí donde los estudiantes aprenden a ser estudiantes y la influencia que puede ejercer el docente es mayor.

Paralelamente a la utilización de estadísticas generales de las escuelas, se utilizó información cualitativa a través de informantes clave (Taylor y Bogdan, 1996) para poder determinar los cursos más "representativos". En este sentido se escogieron cuatro cursos, los cuales reflejaban buena y mala disciplina. Una vez detectados esos cursos se observaron las relaciones de los docentes con sus alumnos, en muchas facetas que a través de la literatura hemos definido: relaciones de poder, legitimación de la autoridad, manejo de la disciplina, reacciones frente a los desarrollos didácticos de los alumnos, etc. Las salas de clase fueron consideradas como las principales unidades de análisis del estudio.

\subsection{Recolección De Información}

Para lograr los objetivos planteados entramos en el campo de trabajo en forma "sigilosa", sutil y no ofensiva, todo esto con el fin de no ser agentes intrusivos que pudieran afectar el desarrollo normal de las actividades en el campo de estudio. Los observadores fueron 4 estudiantes seminaristas de la carrera de pedagogía básica los cuales fueron capacitados en la recolección de información y bajo el ejercicio de su práctica profesional lograron permanecer en la sala de clase todo el primer semestre de 2010, junto a sus docentes guía de aula regular.

Una vez insertos allí, utilizamos técnicas de recolección de información que nos permitieron obtener datos que reflejaran de la manera más fiel la realidad en estudio (Rodríguez, Gil y García, 1999). En esta oportunidad se utilizó la observación participante y no participante, realizándose esta última en la etapa inicial, estableciendo un rapport suficiente para entablar cierta confianza con los potenciales informantes en nuestro proceso de observación (Taylor y Bogdan, 1996). Posteriormente a esto se comenzó con la observación participativa, la cual, como su nombre lo indica, implica una participación directa en las actividades que realizan las personas. Se realizó un total de 160 observaciones (visitas) dentro del período del primer semestre de 2010 las que fueron traducidas como notas de campo. Las observaciones se hicieron todos los días y durante toda la jornada de clase.

Para asegurar que los datos recogidos durante el proceso de observación comprendieran todas las variables y dimensiones contempladas en la investigación, se realizó una "operacionalización" de ésta, lo que permitió diseñar una pauta de entrevista semi-estructurada. La entrevista fue dirigida a los directivos de los centros educativos, los profesores de las aulas en observación y los alumnos de los cursos. El total fue de 16.

Todas estas técnicas de recolección de información. no sólo van en el sentido de tener credibilidad en nuestros datos, sino también para alcanzar a interpretar (Pérez, 2001), es decir, validar lo que se está observando. Es por esto que triangulamos técnicas de recolección de información, durante nuestra permanencia a lo largo de todo el año 2010 y triangulamos los hallazgos y análisis entre todos los colegios observados. Esto nos da mayor poder explicativo de nuestros análisis.

Para los análisis de la información recogida se trabajó la técnica de Strauss y Corbin (2002) conocida como la Teoría Fundamentada, la cual transita en tres grandes momentos: la codificación abierta, la codificación axial y la codificación selectiva. Si bien existe una diferenciación de estos tres momentos, estos no son momentos inconexos, sino más bien se van solapando con el ir y venir de los datos. De esta manera se genera una dinámica entre 
las observaciones y el análisis, generando categorías analíticas que van adquiriendo mayor poder interpretativo. Entonces, lo que busca esta técnica de análisis es ir generando categorías analíticas que nos permitan crear teorías interpretativas de lo que allí está pasando. Esto sólo es posible si realizamos una gran cantidad de observaciones y éstas son sometidas a lo que los mismos autores han denominado como un aspecto fundamental de la metodología, que es la comparación constante (Strauss y Corbin, 2002).

Adicionalmente, y como una complementación especial para nuestros análisis, se utilizó otra técnica de análisis vinculada a la semántica estructural desde la relectura de Sergio Martinic (1992). A partir de esta técnica pudimos dar énfasis a los posibles escenarios, los cuales deberán ir sistematizando las realidades resultantes. Todo esto en aras de un análisis lo más "fino" posible y para no dejar fuera detalles relevantes y estructurantes en la interacción docente-alumno. Finalmente, fueron consideradas las categorías consideradas sobre climas tóxicos y nutritivos realizadas por Aron \& Milicic (1999) para denominar los tipos de climas escolares en observación. Asimismo para caracterizar los tipos de liderazgo de los docentes fueron consideradas las categorías de autoritarios, democráticos o lissez faire (laissez-faire) (Díaz, F., 2002; Ortega et al. 2008) o autocrático, burocrático, paternalista, negociador, libertario (Rodríguez et al. 1998).

\section{Analisis de datos: factores productores de malos climas de aula}

Como una forma de observar las prácticas escolares que implican ciertos grados de violencia, nos situaremos dentro del espacio social de la sala de clase, ya que en ella se confluye todo el accionar de las instituciones escolares, como asimismo es el espacio privilegiado donde se consigue o no el objetivo fundamental de la escuela: el aprendizaje.

Ahora bien, las aulas no son espacios aislados de lo que pasa en la institución misma, pero ellas sí pueden tener una lógica propia sobre todo cuando las escuelas no logran desarrollar un clima organizacional que todos comparten y que se evalúe. Es así como sucede que dentro de las salas de clase pueden tener lugar prácticas escolares muy distintas dentro de un mismo establecimiento escolar. Es más, con un mismo docente en un curso distinto, los resultados pueden ser diametralmente diferentes.

Quizá el lector logre concluir que en estos análisis hay un sobre-enfoque en el actuar del docente, pero esto tiene una lógica: en el nivel de observación en el que hemos decidido trabajar (el aula) lo que hace o no hace el docente es fundamental. En este sentido el peso de los estudiantes de este nivel en la determinación del clima resultante en el aula es menor si lo comparamos con la injerencia o "protagonismo" que pueden tener los estudiantes en la educación media.

A continuación se describen los factores más relevantes para determinar el tipo de clima dentro de las salas de clase. Los factores principales son: estructuración de la clase, el disciplinamiento y el estilo de liderazgo de los docentes.

\subsection{Estructuración de la clase}

Con estructuración de la clase hacemos referencia a dos aspectos esenciales:

La realización de las planificaciones y si éstas se llevan a cabo o no.

De acuerdo al tipo de planificación y si ésta se lleva a cabo o no, puede producirse violencia escolar, tanto directa como indirectamente. Si bien las planificaciones dentro de los discursos de los docentes fueron un elemento importante, en la práctica destacaron por ser considerado un documento "muerto", que es visto como una obligación dentro del ritual del docente, pero 
por lo mismo carece de utilidad práctica en la sala de clase. Dentro de los problemas que se manifiestan cuando la planificación es un ritual sin mayores significados, es que se produce un trabajo inconsistente, con poca preparación y marcado por la improvisación, lo que repercute en la desestabilización del orden dentro de la sala de clase. La siguiente nota de campo ilustra este punto:

"Se puede observar claramente que la clase no está planificada y que las actividades surgen en el momento. A pesar de esto, la profesora mantiene la disciplina en el curso, esto debido a su fuerte carácter y a sus gritos mientras se mueve por la sala".

\subsubsection{Las metodologías, técnicas y estrategias utilizadas por el docente}

La pedagogía que utiliza el docente se verá condicionada por el paradigma de la educación con el cual se identifique. En el estudio de campo se observó que las prácticas educativas no se basan en el constructivismo que mencionan y declaran en las entrevistas. En efecto, las prácticas transitan desde el constructivismo hacia el conductismo y sin embargo es clara la tendencia hacia las prácticas tradicionales. Dentro de las actividades de aula existe una constante de entregar "a todos lo mismo" como un principio que busca la igualdad. La siguiente nota de campo describe este proceso:

“... La profesora hace una clase expositiva en todo momento, donde interpela de vez en cuando a los alumnos..."

Es así que dentro de esta postura no hay mayores posibilidades de hacer la diferencia con los distintos tipos de aprendizaje, ni menos aún deja espacio para las innovaciones pedagógicas. Los docentes sienten especial aversión a los cambios, porque éstos son sinónimo de pérdida de control. Las actividades son bajo una lógica mecánica caracterizada por su falta de reflexión, debate y participación, son actividades poco interactivas bajo un prisma individualista. Es importante hacer notar que esta forma de trabajo prescinde del alumnado. Esto es lo que podemos llamar una "educación sin estudiantes".

\subsection{Disciplinamiento}

Otro factor común en todas las salas de clase observadas es el disciplinamiento, el cual busca enderezar conductas, homogeneizar al alumnado y mantener un orden socialmente aceptado, con el objetivo de aplicar una pedagogía estándar.

A través del estudio se visualizaron distintas técnicas de disciplinamiento, las cuales fueron clasificadas y ordenadas gradualmente. Las primeras son (1) las órdenes, las que obedecen al primer grado y son el nivel más bajo de coerción; a éstas le siguen (2) las amenazas, constituyendo así el segundo grado de aplacamiento de conductas disruptivas y por último el tercer peldaño de esta escala, (3) el castigo. Para ilustrar estas técnicas, a continuación se presenta una nota de campo:

"Durante el transcurso de la clase, la profesora pide orden y silencio. Sin embargo, su manera de pedirlo es gritando y atacando individualmente a cada niño que rompe el esquema disciplinario de la sala de clases".

A medida que la indisciplina aumenta, conjuntamente con ella lo hace esta escala de opresión. Cabe mencionar que estas técnicas son una forma de ejercer violencia por parte del profesor. Sin embargo, estas prácticas tan comunes en los establecimientos son invisibilizadas y legitimadas por gran parte de la comunidad educativa. Claramente las formas que el docente tiene de mantener el orden o recomponerlo son variadas y muchas de ellas tienen formas bastantes perniciosas de conseguir el castigo. Una de las notas de campo que describe esta situación es: 
La profesora comenta "que los niños más inteligentes saldrán a recreo" y los más habladores tendrán que quedarse en la sala. Hay un niño que llora después de este comentario, dice que él no habló en la sala y no terminó su tarea porque no entiende y la profesora lo retó (Nota de campo).

Un aspecto importante a reconocer es que no existe una indisciplina o conducta disruptiva que genere una acción punitiva determinada, sino que todo va a depender del criterio de quien la aplique. En este sentido -y los alumnos se hacen eco de esto-, el castigo y el acto del disciplinamiento son muchas veces arbitrarios y ambiguos, lo que ciertamente desconcierta y le hace perder legitimidad entre los estudiantes. En este sentido, el disciplinamiento no tiene un carácter formador sino sancionador. La pedagogía de estos profesores no da cabida a que la disciplina sea un acto que conlleve aprendizaje sino sólo es sancionador de las conductas que no son aceptadas, pero con este afán punitivo lo que hace es mantener las diferencias entre los alumnos; entre aquellos que se normalizan y a los que hay que "normalizar", los que son objetos de castigo. Entonces como la disciplina es un castigo constante, el docente se vale de muchas maneras para sancionar, pasando del uso de su propio cuerpo (gritar, golpear) hasta el uso de la calificación como mecanismo coercitivo y clasificador de los estudiantes.

\subsection{Estilos de liderazgo, poder y autoridad docente}

El cómo el docente se posiciona dentro de la sala de clase influirá mucho en la forma de relacionarse con sus estudiantes y en este sentido la pedagogía que desarrolle y la distancia que establezca.

El tema del poder y la autoridad del docente no parece ser un aspecto que los estudiantes discutan, sino que existe una atribución automática que los docentes tratan de mantener bajo varias formas de liderazgo. Por ejemplo, se pudo observar en las salas de clase que existen docentes que imponen visiones y formas de proceder, las cuales no tienen ningún principio democrático: aquí el alumno sólo tiene que acatar "lo que se le dice".

“... o sea, tú como profesor, por sobre todas las cosas tienes que imponerte, porque si tú no te impones como profesor tu clase, simplemente, no se lleva a cabo... es que tú tienes que imponerte ante tu curso, tan simple como eso, nada más... tú estás en la sala de clases, tú eres la autoridad y se hace lo que Tú dices..." (Entrevista docente).

Para lograr esto, el docente construye todo un aparataje o mecanismo que facilita esta posición absoluta de autoridad, como lo es el disciplinamiento constante, una postura intransigente, una voz y postura corporal de intimidación, sanciones constantes. Aquí la asimetría es clara y el docente tiene el monopolio de la palabra y decisión. Si bien éste es un tipo común de docente, el cual es considerado como "buen profesor", no es el único que pudo ser observado. Dentro de las observaciones de campo, se pudo caracterizar distintos tipos de docente. Por ejemplo, hay docentes con cierta cercanía con los estudiantes y otros que son de carácter más ambiguo o inestable, pero todos ellos tratan el tema de la autoridad como una cuestión vital. Para algunos de ellos la atribución de la autoridad es concedida de forma legítima, en otros se impone. Como menciona un docente:

"si el niño no te hace caso, tú estás mal, a como dé lugar el niño tiene que hacerte caso. ¡YA! ¡Tiene que hacerte caso! Porque el curso se va a dar cuenta”. (Entrevista docente). 
Si bien la autoridad y el tipo de liderazgo que de ella se derivan son concebidos como una forma de alcanzar ciertos objetivos educativos, también se los puede visualizar como un mecanismo netamente de control, y cuando esto es así estamos en presencia de un autoritarismo escolar. Lo más preocupante es que dentro de los discursos de los docentes esto es visto como algo positivo y deseable; la idea del mantenimiento del orden es considerado como un bien que sobrepasa el tipo de aprendizaje que se puede lograr con los estudiantes. En definitiva, existe una legitimación de las formas autoritarias del ejercicio docente.

\section{Distintos escenarios posibles}

Los tres factores antes mencionados son considerados como ejes principales en la estructuración de los climas que se pueden establecer al interior de la sala de clase. A partir de ellos, y considerando las diferencias en el manejo de la disciplina escolar que los docentes pueden desarrollar, surgen distintos escenarios o realidades que es preciso rescatar y conceptualizar. Por lo tanto, como una forma de conceptualizar las realidades observadas se desarrolla a continuación la descripción de diferentes climas de aula resultantes de la interacción entre la estructura de las clases que un docente desarrolla y el nivel de disciplina que el docente logra ${ }^{1}$. Dentro de estos escenarios es fácil visualizar la violencia implícita o explícita que es producto directo de las relaciones educativas.

\section{Figura $\mathbf{N}^{\circ} 1$}

Fuente: Elaboración propia.

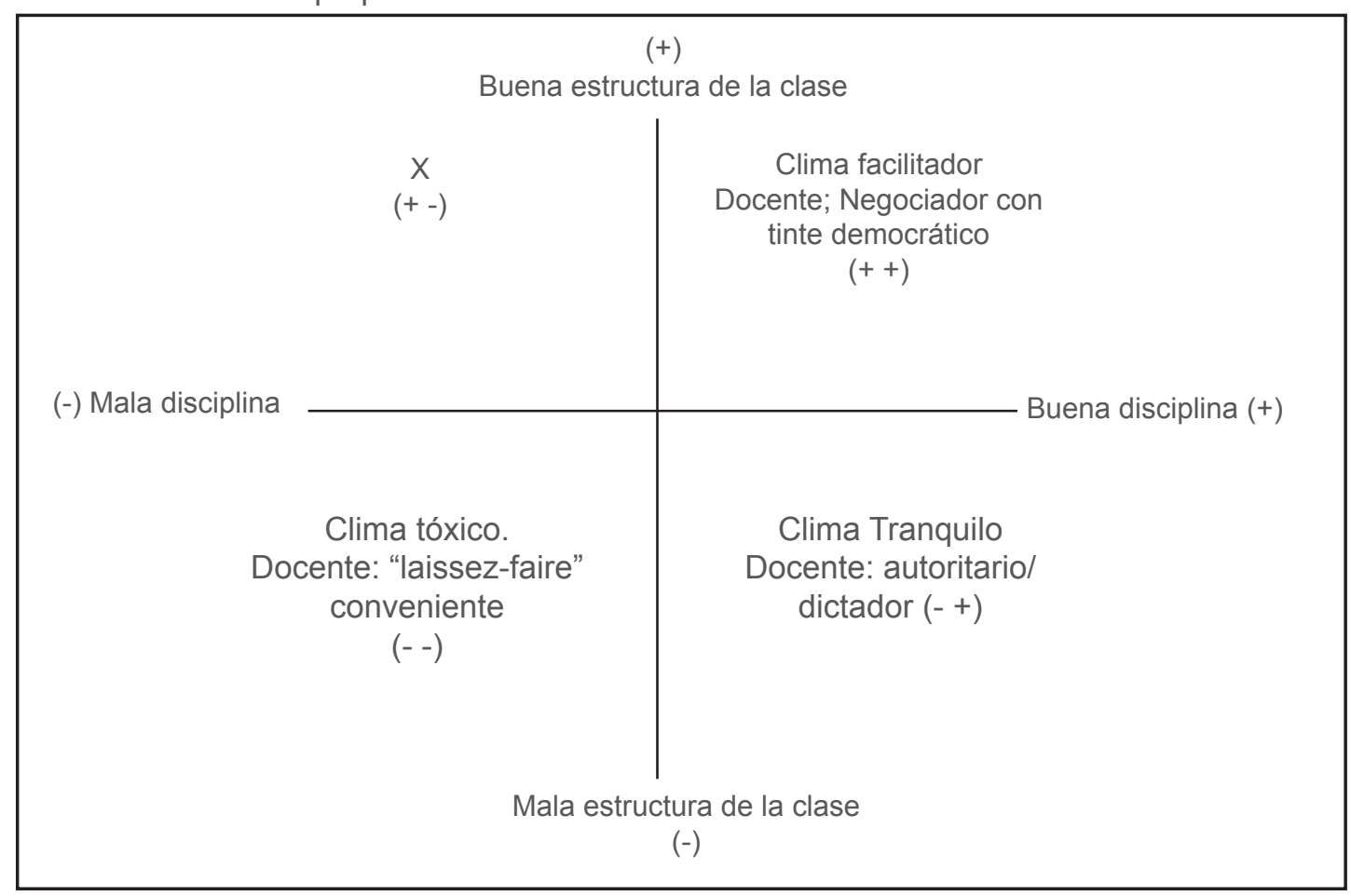




\subsection{Tipo de clima}

La figura $\mathrm{N}^{\circ} 1$ evidencia cuatro diferentes climas dentro de las salas de clase que son producto del cruce de los dos ejes principales que hemos elegido. A partir de esta metodología es posible describir tres de los cuatro escenarios posibles. Uno de ellos no tiene correlato con la realidad observada. Esta realidad ha sido denominada "X", ya que no es posible de describir y no tiene sentido lógico. Los restantes tres escenarios son buenos descriptores de las realidades observadas.

\subsubsection{Escenario buena estructura/ buena disciplina: clima facilitador}

Si bien se observa que hay una buena estructura y existe más aprendizaje, tal como lo clasificaron Aron \& Milicic (1999) este tipo de clima no es perfecto. Un clima facilitador, como su nombre lo indica, proporciona instancias para que se lleve a cabo la clase en su totalidad y que de ésta se obtengan los resultados esperados, tanto a nivel cognitivo como social. En este sentido, la percepción que los alumnos tienen del clima que se vive en su sala de clase es el de un ambiente acogedor que sigue las normas y reglas impuestas por el docente.

El clima facilitador se caracteriza por la inexistencia de tiempos muertos, debido a que existe una planificación de la clase y posterior realización de ésta. A pesar de ser un clima aparentemente "bueno", existen momentos en los que el docente debe ejercer un fuerte disciplinamiento, debido a que las acciones disruptivas de los alumnos, sobrepasan los límites permitidos por él. A partir de las observaciones realizadas es posible definir a este tipo de docente como un profesor negociador con tinte democrático.

“... bueno de hecho una clase... donde un niño no opine o no se exprese no es mi ideal, o sea mi ideal es sí mantener un orden... mantener un ritmo de trabajo, pero en el que ellos también puedan interactuar, puedan decir lo que piensan, puedan levantar la mano "tía tal cosa" aportar a la clase... yo quiero que ellos aprendan y necesito tener un ambiente de trabajo agradable o sea donde ellos se sientan cómodos de expresar lo que quieran..." (Entrevista docente).

Si combinamos este tipo de docente con este clima de aula facilitador, se produce en los educandos un aprendizaje en todas las dimensiones, es decir, en el ámbito cognitivo y valórico, ya que se toma en cuenta la transversalidad estipulada en el currículo, plan de estudios formal, se respetan los ritmos de aprendizaje utilizando actividades diferenciadas y se da una relación afectiva entre los distintos actores, debido a la buena y fluida comunicación entre éstos. Además este último punto facilita que el comportamiento de los alumnos sea "relativamente bueno" dentro de la sala de clase, es decir, alumnos disciplinados, con un cierto grado de autonomía y con capacidad de diálogo.

\subsubsection{Escenario alto nivel de disciplinamiento/clases no estructuradas: clima tranquilo}

Este tipo de clima se caracteriza por el orden establecido en el ambiente de clase, donde los niños generalmente aguardan sentados y en silencio el contenido que el profesor les entrega diariamente. Aquí los niños son sumisos y obedecen a lo que el profesor impone, debido a que el trato es áspero y riguroso, con gritos y castigos, asemejándose y acercándose más a un instructor que a un profesor, controlando todas las acciones, desde cómo sentarse, hasta cómo poner atención.

Un clima tranquilo se caracteriza por la existencia de tiempos muertos a raíz de la no utilización de las planificaciones. Sin embargo, mayoritariamente estos espacios no son instancias de "desorden" debido a la férrea disciplina que opera en ellos. Esta prevención del "desorden" se realiza a través de gritos y amenazas, pero cuando esto falla y afloran las conductas disruptivas, se hace uso de técnicas duras de represión. El profesor que opera aquí es el autoritario/ 
dictador, que al combinarse con este clima genera alumnos disciplinados, sumisos, irreflexivos y con escasa autonomía; el nivel de violencia que se produce entre pares es bajo ya que las instancias de interacción son limitadas. Sin embargo, en la relación docente/alumno existe un alto nivel de violencia ejercida por el profesor, generando una relación distante debido al miedo que estos últimos demuestran por la rudeza con la que el docente ejecuta sus acciones.

El trato brusco e inflexible del docente genera paulatinamente miedo en los niños, el cual poco a poco se va apoderando de ellos, haciéndose patente en todas sus acciones. La consecuencia de este miedo entonces es un orden casi "militar" debido a la fuerte represión ejercida por el docente, ya que ante cualquier emancipación aquélla actúa de forma punitiva. El trabajo en clases se ve favorecido por este ambiente de calma, ya que el orden existente en él permite mantener un buen ritmo de trabajo. Es así como el buen ritmo de la clase y la disciplina arraigada a la fuerza generan un ambiente propicio para el traspaso de conceptos y una pedagogía de orden tradicional, generando un aprendizaje impuesto, teórico y sin apropiación en los niños.

Este escenario resulta interesante por cuanto éste es un clima que es deseado por los docentes. En muchas de las entrevistas fue vista como la situación que facilita el poder llevar la clase a cabo.

“...siempre se está hablando de la indisciplina, y si tú no tienes control en tu sala de clases, si tú no controlas la disciplina, tú podrás tener una planificación muy bonita, pero lo primordial en una clase es la disciplina" (Entrevista docente).

Ciertamente uno de los elementos clave para esta discusión es saber cuáles son los objetivos y cuáles de ellos son los más importantes. A este respecto tenemos que muchos de los objetivos que los docentes se trazan están muy estrechamente relacionados con las presiones institucionales del "buen rendimiento", no importando demasiado el qué aprenden ni el cómo lo hacen.

“...el año pasado lo viví y fue una experiencia bien tensa para los niños, tensa para el profesor... Están trabajando con el jefe de DAEM (jefe comunal de educación), ahí vienen a trabajar todos los días" (Entrevista docente).

Este fenómeno es el que podemos interpretar como la cosificación de la educación que trabaja más un conocimiento alienado que uno significativo para los estudiantes, donde lo importante es que sepan reproducir un conocimiento dado y que sepan rendir un examen.

\subsubsection{Escenario mala estructura/ indisciplina: clima tóxico}

Tomando en cuenta las características de este clima, se lo ha catalogado cómo tóxico (Aron \& Milicic 1999), ya que el mismo no es propicio para el aprendizaje tanto conceptual como actitudinal ni genera espacios para una adecuada convivencia, ya sea entre docente-alumno o alumno-alumno. La siguiente situación refleja este punto:

"Al que va terminando la actividad de apresto se le va entregando una fotocopia para que la pinten" (Comentario del observador (CO): No logro ver ninguna intención pedagógica en esta actividad de pintar, sin duda creo que es sólo para matar el tiempo, además de ser actividades improvisadas pero a pesar de todo esto, de la improvisación y la no planificación, se mantiene el orden en la sala debido a la represión ejercida por la profesora en todo momento y orden de cosas) (Nota de campo).

Un clima tóxico se caracteriza por la presencia excesiva de tiempos muertos debido a que no se cumple con la puesta en marcha de las planificaciones o, directamente las clases no son planificadas por lo que cunde el caos y la indisciplina se hace patente, lo que indiscutiblemente genera una inadecuada convivencia entre los actores que interactúan en el aula. Este clima, al igual que los dos anteriores, debe contar con un profesor característico, en este caso el 
laissez-faire conveniente que al operar tiene como resultado escasos aprendizajes tanto conceptuales como valóricos, y cuando se producen son significativamente bajos. La siguiente nota de campo ilustra este proceso:

Cuando los niños terminan y no saben qué más hacer y le preguntan a la profesora y a mí “¿Qué hacemos ahora?" Algunos empiezan a comer y a conversar. La profesora los manda a dibujar lo que ellos quieran. (C.O.: claramente) la profesora no tenía ninguna actividad en mente, por eso los mandaba a hacer, más bien dibujar, lo que quisieran. El ruido empezó a crecer lentamente, pero la profesora no prestó atención a ello y los dejó, y aunque sólo bastara un grito para que callaran y se ordenaran, sin embargo no lo hizo".

Estas prácticas generan un clima de desorden, donde el docente interviene intermitentemente con gritos y amenazas, los cuales al no ser cumplidos generan incredulidad por parte del alumnado. Estas técnicas de disciplinamiento no cumplen con su objetivo final, ya que no suprime (suprimen) estas acciones disruptivas, sino que las aquietan sólo por el momento.

Estos tipos de clima no se dan en cualquier curso ni escuela. Por lo general esta situación se da en los cursos catalogados como "malos" donde la predisposición, ya sea de los estudiantes como de los profesores, es negativa. Dentro de este esquema es donde se reproduce el déficit que los alumnos han acumulado a través de sus historias tanto familiares como académicas, y lamentablemente las escuelas no responden ante esta situación sino por medio de la sanción y las bajas expectativas. Claramente éste es un tipo de violencia institucional que a través de sus prácticas pedagógicas produce estudiantes de bajas trayectorias académicas.

\section{Discusiones preliminares}

Las instituciones educativas pueden provocar cambios significativos en sus estudiantes, cuando éstas tienen una organización clara y eficaz (UNESCO, 2002, 2008; Murillo, 2003; Maureira, 2006). Sin embargo, cuando éstas carecen de una lógica u organización interna consciente, dejan que las variables contextuales operen sin mayores dificultades. Esto último es un problema cuando tenemos estudiantes de bajo capital cultural y envueltos dentro de una cultura de la contingencia.

Enfocándonos en la idea de que la institución escolar genera sus propios microclimas, éstos pueden ser compartidos y trabajados de forma consciente o pueden generarse con una lógica independiente que no siempre está vinculada a la escuela y no tiene un proyecto educativo claro. Éste es el caso de los escenarios que hemos detectado en la investigación. Las acciones que van desde la apatía pasando por el desorden hasta la violencia que presentan los estudiantes irrumpen en determinados contextos, de los cuales, en estos casos, el docente era el gran organizador. Por lo tanto, uno de los elementos importantes a considerar es: primero que la escuela tiene lógicas internas y segundo que éstas pueden existir de una forma consciente y planificada o, por el contrario, sólo producto de prácticas individuales y erráticas.

Ahora bien, la violencia escolar depende de la mirada que se privilegie y de quien la legitima. Dentro de los escenarios observados, el docente es el eje articulador de todo lo que pasa en la sala de clase y en este sentido la violencia observada es sólo la que producen los estudiantes o ciertos estudiantes, los cuales a su vez son sancionados por ciertas conductas. Claramente el énfasis está puesto en el cumplimiento o no de una determinada normativa, y por consiguiente las acciones que se sancionan son sólo las que entran dentro de este parámetro (INJUV, 1999). Como el ojo rotulador aquí es el docente, éste no logra ver su propia acción bajo la lógica del rompimiento de una norma de convivencia. Es más, él mismo se piensa muchas veces como el que siempre la está buscando y los alumnos tratando de romperla. 
De esta forma se comienza con una lucha permanente de tratar de mantener un "orden" que se desea, pero éste no surge como un consenso sino que aparece como un acto por antonomasia: "es este tipo de orden el que nos permite conseguir los resultados deseados" mencionan los docentes. El objetivo supremo de mantener la buena convivencia, la que se entiende como el mantenimiento del orden y la disciplina impide que los docentes den protagonismo a los estudiantes, que éstos trabajen en grupos, que interactúen con el docente, porque siempre está el miedo a la pérdida de control. Esto último explica con mucha claridad el por qué los docentes emplean un tipo de liderazgo asentado en el autoritarismo que genera un sinfín de resistencias en todos los ámbitos de acción, como por ejemplo en el cambio de la pedagogía. En este sentido los modelos de comportamiento que se generan y legitiman dentro de la sala de clase no permiten la generación de conocimiento sino más bien la reproducción de éste. Por lo tanto, al examinar el cómo se construye el conocimiento dentro de la sala de clase nos estamos preguntando por los tipos de autoridad (Crawford, 2008; Pace \& Hemmings, 2007) que se construyen y legitiman en la vida social del aula.

Este liderazgo autoritario muchas veces desconoce a los estudiantes sus necesidades y formas de aprendizaje, y más aún muchas veces los estudiantes perciben y sienten discriminación al interior de las salas de clase, que van desde cuestiones especificas como la ridiculización hasta la negación de la legitimidad de las diferencias (Mena y otros, 2006). En este sentido la propuesta de Casassus $(2007)$ y la UNESCO $(2002,2008)$ de incorporar factores emocionales en los climas de aula para mejorar la predisposición y los resultados de aprendizaje queda relegada a un segundo plano, ya sea porque esto no es considerado importante ${ }^{2}$ o porque simplemente tales resultados no se miden. Parafraseando a Sús (2005) el tema del disciplinamiento constante por parte de" los docentes pone de relieve "los aprendizajes que la misma institución genera, y denuncia la fractura en el vínculo docente-alumno que se visibiliza en el encuentro pedagógico (pág. 985).

Es importante destacar que la intervención que hacen los docentes cuando quieren restablecer el orden es bajo la óptica conductista, es decir, sólo se genera una cierta acción que el docente espera que el alumno reconozca como una señal de desaprobación. Por ejemplo, cuando los estudiantes están desordenados el docente inmediatamente los sanciona, pensando que esa es la acción que permitirá la enseñanza al alumno pero en ningún caso se detiene a explicar, a dar herramientas a los estudiantes para que se produzca un proceso de aprendizaje real. Es por eso que cuando el docente no está vigilando, el estudiante retoma la indisciplina. Por lo tanto, la forma de conseguir la buena disciplina en los estudiantes no es a través del desarrollo de valores, autonomía o autodisciplina, sino a través de la lógica del premio y castigo, sin dar una explicación o razonamiento por parte del docente.

Es aquí donde las prácticas escolares son una fuente de violencia hacia los estudiantes, los cuales entran bajo el ejercicio de enmarcación fuerte (Bernstein, 1994) y agresión simbólica (Bourdieu y Passeron, 2001). El acento en el ejercicio disciplinario de los docentes no siempre tiene una lógica clara, sino como es producto de las prácticas rutinarias o inercia (UNESCO, 2008) está compuesto por muchos elementos poco claros y ambiguos. En este sentido, el castigo y las reacciones que tienen los docentes son de carácter errático, inconsistente e inconstante, lo que por supuesto genera menos posibilidad de aprendizaje de parte de los alumnos. Este elemento de inconsistencia refleja la escasa formación profesional de los docentes en los manejos de grupo y por este motivo la adopción de modelos de autoridad pedagógica son producto de la imitación y las viejas prácticas.

Las inconsistencias en el actuar pedagógico y los malos objetivos del disciplinamiento (o carencia) que tienen los docentes son espacios abiertos a las arbitrariedades del docente y 
a las disrupciones de los estudiantes. Son estos dos elementos que hacen visible y provocan cierto clima de aula que no favorece el aprendizaje escolar en todas sus dimensiones, ya sea en la tan anhelada mejora de los indicadores como en la tan discutida valoración de los aspectos transversales del currículo.

Por lo tanto, la escuela es un espacio social donde se dan una serie de interacciones y creencias que van desencadenando y manteniendo micro-estructuras. A partir de esta construcción se legitiman ciertas prácticas educativas y se invisibilizan otras.

Ciertamente la discusión sobre lo que se puede esperar y desear de la escuela. Sin embargo es necesario preguntarse por la dirección social y los objetivos educativos que presionan los resultados educativos y la mejora (Wrigley, 2003). Esto fue un factor importante para poder entender las prácticas educativas de los docentes y su deseo de mantener un ordenamiento estricto de los estudiantes, ya que bajo su lógica es ésta una de las únicas formas de poder "enseñar" a los estudiantes y que efectivamente consigan los resultados que la política educativa, la institución escolar, el docente y las familias desean. La presión por los indicadores o los "buenos resultados" se ve reflejada en la constante preocupación por el SIMCE que se ha transformado en una de las preocupaciones primordiales y constituyentes de las prácticas escolares. La práctica repetitiva y el entrenamiento constante de los estudiantes producen cosificación educativa: ya no es importante qué tanto aprendan y qué aprendan los estudiantes, sino sólo que tengan un puntaje alto.

Por lo tanto, al pensar la violencia escolar no sólo tenemos que observar el cómo las instituciones educativas y las acciones pedagógicas de los docentes generan la violencia escolar entre los estudiantes, sino también el cómo aquéllas la generan. Las prácticas educativas aquí observadas se centran más en los hechos y no en las causas que generan indisciplina y violencia escolar. Por ejemplo la escuela reacciona cuando se trasgrede un determinado reglamento o normativa, pero no reacciona con respecto a la tensión que la produjo.

\section{Bibliografía}

Araos, C. y Correa, V. (2004). La escuela hace la diferencia. Aproximación sociológica a la violencia escolar. Seminario de titulación, Fundación Paz Ciudadana e Instituto de Sociología PUC, Santiago, Chile.

Arón, A. y Milicic, N. (1999). Clima social escolar y desarrollo personal. Un programa de mejoramiento. Santiago, Chile: Editorial Andrés Bello.

Bernstein, B. (1994). La estructura del discurso pedagógico: clases, códigos y control. Madrid, La Coruña. Morata; Paideia.

Bourdieu, P. y Passeron, J.C. (2001. La reproducción: elementos para una teoría del sistema de enseñanza. Madrid: Editorial Popular S.A.

Casassus, J. (2007) La educación del ser emocional. Santiago: cuarto propio. (Editorial Cuarto Propio). Segunda Edición.

Cornejo y Redondo. (2001). El clima escolar percibido por los alumnos de enseñanza media. Una investigación de algunos liceos de la Región Metropolitana. En Revista Última Década $N^{\circ} 15$, CIDPA, Viña del Mar.

Crawford, T. (2008). Winning the epistemological struggle: Constructing a cultural model of shared authority in an elementary classroom. Teachers College Record, Vol. 110, № 8. 
Fierro, Ma. C. (2005). El problema de la indisciplina desde la perspectiva de la gestión directiva en escuelas públicas del nivel básico. En Red de Revistas Mexicana de Investigación Educativa Redalyc. Año/Vol. 10 N²7, México.

Furlan, A. 2005). Problemas de indisciplina y violencia en la escuela. En Red de Revistas Mexicanas de Investigación Educativa Redalyc. Año/Vol. 10 N²6, México.

Gallego, G. y Anton H. (2008). ¿Aulas conflictivas? La opinión del profesorado. Editorial Dykinson.

García, M. y Madriaza, P. (2005) Sentido y Sinsentido de la Violencia Escolar. Análisis Cualitativa [sic] del Discurso de Estudiantes Chilenos. Psykhe, vol.14, no.1, pp.165-180. ISSN 0718-2228.

Giroux, H. (1990) Profesores como intelectuales. Hacia una pedagogía crítica del aprendizaje. Barcelona, España: Ediciones Paidós Ibérica S.A.

Guerrero, P. (2005). Estudio de la resistencia de los profesores a una estrategia para el desarrollo de la creatividad en tres unidades educativas. En Revista Psykhe, Vol. 14, № 1.

Henríquez, G. y Barriga, O. (2005) (en prensa). El Rombo de la Investigación. Cinta de Moebio. No. 22. Facultad de Ciencias Sociales, Universidad de Chile. (http://www.moebio.uchile.cl)

INJUV (1999). Conflicto y mediación en el medio escolar. Centro de investigación y desarrollo de la educación CIDE.

Magendzo, A. y Donoso, P. (2000). Cuando a uno lo molestan. Un acercamiento a la discriminación en la escuela. Santiago: Lom-PIIE

Martinic, S. (1992). Análisis estructural: presentación de un método para el estudio de lógicas culturales. Centro de Investigación y Desarrollo de la Educación, CIDE. Santiago.

Maureira, O. (2006). Dirección y eficacia escolar, una relación fundamental. Revista Electrónica Iberoamericana sobre Calidad, Eficacia y Cambio en Educación REICE, Vol. 4, No. 4e.

Mena M., Milicia N., Romagnoli C. y Valdés A. (2006). Potenciación de políticas públicas de convivencia escolar. Propuesta Valora UC, concurso de políticas públicas UC. En; www. educomputacion.cl/content/view/73/62/ Recuperado (19/02/2010).

Ministerio del Interior (2007). Encuesta de violencia en el ámbito escolar 2005-2007. Ministerio del Interior-Adimark. http://www.seguridadpublica.gov.cl/files/presentacion_envae_2007final. pdfMurillo, J. (2003). Una panorámica de la investigación iberoamericana sobre eficacia escolar. Revista Electrónica Iberoamericana sobre Calidad, Eficacia y Cambio en Educación REICE, Vol. 1, No. 1.

Ortega, P., Mínguez, R. y Saura, P. (2005). Conflictos en las aulas. Editorial Ariel. $2^{\circ}$ edición, Barcelona. España.

"Pace, J. \& Hemmings, A." (2007). Understanding authority in classrooms: A review of theory, ideology and research. Review of Educational Research. Vol. 77, N 1.

Pérez, G. (2001). Investigación cualitativa: retos e interrogantes. La Muralla, Madrid. Vol 2. (Vol.)

PNUD (1998). Las paradojas de la modernidad. Programa de las Naciones Unidas. Santiago. Rodríguez, y otros. (inicial de nombre de pila de Rodríguez? Si se cambia hay que cambiarlo todas las veces que lo cita en el texto) (1999). Metodología de la investigación cualitativa. Ed. Aljibe. Málaga. España. 
Saavedra, E., Villalta, M. y Muñoz, M. (2007). Violencia escolar: la mirada de los docentes. Revista Límite. Revista de filosofía y psicología. (Vol.) 2, № 15 pp 39-60.

Strauss y Corbin. (2002). Bases de la investigación cualitativa. Técnicas y procedimientos para desarrollar la teoría fundamentada. Bogotá. Colombia. CONTUS. $2^{\circ}$. Ed.

Sús, M. (2005). Convivencia o disciplina; ¿Qué está pasando en la escuela? En Red de Revistas Mexicana de Investigación Educativa Redalyc. Año/Vol. 10, №27, México.

Taylor y Bogdan (1996). Introducción a los Métodos Cualitativos de Investigación. Ed. Paidós.

Torres, J. (1992). El currículum oculto. Editorial Morata, Madrid.

Trianas, Sánchez y Muñoz (2001). Educar la convivencia como prevención de violencia interpersonal: perspectiva de los profesores. Revista Interuniversitaria de formación del profesorado, N 41, pp 73-93. Universidad de Zaragoza, España.

UNESCO (2002). Estudio cualitativo de escuelas con resultados destacables en siete países latinoamericanos. UNESCO-Santiago.

UNESCO y MINEDUC (2005). Primer estudio nacional de convivencia escolar: la opinión de estudiantes y docentes. Ejecutado por el Instituto de Evaluación y Asesoramiento Educativo [Idea Chile]. Disponible en versión digital en http://www.comisionunesco.cl/Unesco/Documentos/ educacion/informe_ejecutivo_estudio.pdf (Recuperado el 2 de junio de 2009).

UNESCO (2008a). Convivencia democrática, inclusión y cultura de paz. Lecciones desde la práctica educativa innovadora en América Latina. Equipo Innovemos, OREALC/UNESCO Santiago.

UNESCO (2008b). Eficacia escolar y factores asociados en América Latina y el Caribe. OREALC/ UNESCO Santiago, LLECE.

Valles, M. (2000). Técnicas cualitativas de investigación social. Reflexiones metodológicas y práctica profesional. Ed. Síntesis, España.

Villalta, M., Saavedra, E. y Muñoz, M. (2007). Pasado a llevar. La violencia en la educación media municipalizada. En Revista Estudios Pedagógicos XXXIII, Nº 1.

Vieytes, R. (2004). Metodología de la investigación en organizaciones, mercado y sociedad. epistemología y técnicas. Ed. De las Ciencias. Argentina.

Wrigley, T. (2003). Is school effectiveness anti-democratic? British Journal of Educational studies, Vol. 51, Nº 2 pp 89-112.

Yoneyama, S. y Naito A. (2003). Problem with paradigm: the school as a factor in understanding bullying (with special reference to Japan). British Journal of Sociology of Education, Vol. 24, $N^{\circ} 3$. 


\section{Notas}

${ }^{1}$ Según Martinic (1992) "Al cruzar dos ejes de calificación se forman cuatro realidades en las cuales dos adquieren un valor ambivalente ya que ellas integran el término positivo de un código y el término negativo de otro, la tercera es totalmente positiva y la cuarta totalmente negativa" (pp. 27).

${ }^{2}$ La idea de la transversalidad es una cuestión que no se trabaja en las salas de clase. La idea de que están en forma implícita en el currículo las hace aparecer como una cuestión que se da por sí sola y que no requiere de implementación consciente.

La información recabada en esta investigación fue producto del trabajo de seminario de título de los alumnos Daniel Ayala, Marcela Bustamante, Carol Jara y Yasna Sandoval quienes optaron al título de Profesor de Educación Básica, Universidad de Concepción, Campus Los Ángeles, Chile.

Fecha de recibido: 04/02/2013

Fecha de aceptación: 03/06/2013

* Ph.D. (Cand.) en Educación, Universidad de Sydney, Australia. Master en Investigación social y desarrollo, Universidad de Concepción, Chile. Sociólogo, Universidad de Concepción, Chile. Docente del Departamento de Teoría, política y fundamentos de la educación, Escuela de Educación, Universidad de Concepción, Chile. 